\title{
Relaxation dynamics of disordered Ising-like models
}

\author{
R.R.Levitskii, R.O.Sokolovskii \\ Institute for Condensed Matter Physics \\ of the National Academy of Sciences of Ukraine, \\ 1 Svientsitskii Str., 79011 Lviv, Ukraine
}

Received October 11, 1998

\begin{abstract}
Two-site cluster approximation to Glauber's master equation is developed for lattice spin systems with substitutional chaos. Dynamic pair correlation functions of disordered Ising and Mitsui models are calculated. The agreement between the theory and the results of dielectric measurements in ferroelectric crystals $\mathrm{Cs}\left(\mathrm{H}_{1-x} \mathrm{D}_{x}\right)_{2} \mathrm{PO}_{4}, \mathrm{Rs}_{1-x} \mathrm{dRs}$ is remarkably good.
\end{abstract}

Key words: Ising model, Mitsui model, kinetic equation, order-disorder ferroelectrics, dynamic permittivity

PACS: $64.60 . \mathrm{Cn}$

\section{Introduction}

Being the model of critical phenomena in many different objects an Ising model compels the attention during many years. Within the framework of quasispin models successful efforts are undertaken to describe a variety of ferroelectric phenomena in different ferroelectric crystals. In this paper we study the kinetics of Ising and Mitsui models (within master equation [1] approach) in order to describe the dynamic properties of partially deuterated ferroelectrics of order-disorder type. We develop a two-site cluster approximation (TCA) for strong short-range interactions present in such crystals. The long-range interactions will be taken into account within the mean field approximation.

\section{The model and the approach}

We consider the spin system on the Bravais lattice which is described by the Ising Hamiltonian

$$
H=-\sum_{i} \kappa_{i} S_{i}-\frac{1}{2} \sum_{i j} K_{i j} S_{i} S_{j}
$$


$H$ describes the set of Ising spins with the pair exchange interaction $K_{i j}$ in the sitedependent field $\kappa_{i}$. Variables $\left\{S_{i}\right\}\left(S_{i}= \pm 1 ; i=1 \cdots N, N\right.$ is a number of the sites) represent states of crystal structure elements that have got two equilibrium positions. These structure elements carry electric dipole moments, and their ordering leads to the emerging of spontaneous polarization in these crystals. Deuteration brings disorder in the system, because in a partially deuterated crystal there are two types of dipoles $\left(\mathrm{O}-\mathrm{H} \cdots \mathrm{O}\right.$ and $\mathrm{O}-\mathrm{D} \cdots \mathrm{O}$ hydrogen bonds in the case of $\mathrm{CsH}_{2} \mathrm{PO}_{4}$ crystal or $\mathrm{OH}$ and $\mathrm{OD}$ hydroxyl groups in the Rs crystal). This requires considering a disordered Ising model, in which the quantities $\kappa_{i}, K_{i j}$ are random,

$$
\kappa_{i}=\sum_{\alpha} \kappa_{i \alpha} X_{i \alpha}, K_{i j}=\sum_{\alpha \beta} K_{i \alpha, j \beta} X_{i \alpha} X_{j \beta}
$$

where the set of variables $\left\{X_{i \alpha}\right\}$ describes the sort configuration $\left(X_{i \alpha}=1\right.$, if the site $i$ is occupied by spin of the sort $\alpha$, otherwise $X_{i \alpha}=0 ; \alpha=1$ for $\mathrm{H}$ ion and $\alpha=2$ for $\mathrm{D}$ ion). The true parameters of the Hamiltonian (2.1) are $\kappa_{i \alpha}, K_{i \alpha, j \beta}$. Since long range interactions can be taken into account in the mean field approximation (these simply lead to the emerging of effective field term in the fields $\kappa_{i \alpha}$ ) $[2,3]$ here we suppose the pair interactions to be short-range

$$
K_{i \alpha, j \beta}=K_{\alpha \beta} \pi_{i j} ; K_{\alpha \beta}>0 ; \pi_{i j}= \begin{cases}1, & \text { if } j \in \pi_{i} \\ 0, & \text { otherwise }\end{cases}
$$

where $\pi_{i}$ denotes the set of the nearest neighbours of the site $i$ (the first coordination sphere).

We shall consider the quenched system (frozen sort configuration) and investigate its dynamics based on Glauber's master equation [1] for the distribution function $\rho(\{S\}, t)$ :

$$
\begin{aligned}
\frac{\mathrm{d}}{\mathrm{dt}} \rho(\{S\}, t)= & -\sum_{i} W_{i}\left(\cdots S_{i} \cdots\right) \rho\left(\cdots S_{i} \cdots, t\right) \\
& +\sum_{i} W_{i}\left(\cdots-S_{i} \cdots\right) \rho\left(\cdots-S_{i} \cdots, t\right) .
\end{aligned}
$$

Following [1] the probability per unit time that the $i$ th spin flips from the value $S_{i}$ to $-S_{i}$ is assumed to be

$$
W_{i}\left(\cdots S_{i} \cdots\right)=\frac{1}{2 \tau_{i}}\left(1-S_{i} \tanh \beta \varepsilon_{i}\right)
$$

where $\beta=1 / k_{\mathrm{B}} T$,

$$
\varepsilon_{i}=\kappa_{i}+\sum_{j} K_{i j} S_{j}
$$

is a local effective field acting on the $i$ th spin. In this approach the dissipative properties of the spin subsystem is caused by its interaction with the other (for example, phononic) subsystems which play the role of a heat bath. The quantity $\tau_{i}$ has the dimension of time and defines the time scale of relaxational phenomena. We assume the quantity $\tau_{i}$ to be locally sort-dependent: 


$$
\tau_{i}=\sum_{\alpha} \tau_{\alpha}^{0} X_{i \alpha}
$$

Relations (2.4) and (2.5), (2.6) lead to the following kinetic equations for the average value of spin:

$$
D_{i, t}\left\langle S_{i}\right\rangle_{H, t}=\left\langle\tanh \beta \varepsilon_{i}\right\rangle_{H, t}
$$

where

$$
\langle(\cdots)\rangle_{H, t}=\operatorname{Sp}_{s} \rho(\{S\}, t)(\cdots) ; D_{i, t}=1+\tau_{i} \frac{\mathrm{d}}{\mathrm{dt}} .
$$

The system is disordered, therefore in order to calculate experimentally measured quantities you have to perform an averaging over the sort configurations. For example, the polarization of a partially deuterated H-bonded ferroelectric with the Hamiltonian (2.1) is defined as

$$
P(t)=\frac{1}{V} \sum_{i} \sum_{\alpha} \mu_{\alpha}\left\langle\left\langle S_{i \alpha}\right\rangle_{H, t}\right\rangle_{x} ; S_{i \alpha}=S_{i} X_{i \alpha}
$$

where $V$ is a volume of the system, $\mu_{\alpha}$ the dipole electric moment associated with the quasispin of sort $\alpha$ (proton or deuteron), $\langle\cdots\rangle_{x}$ denotes the averaging over sort configurations with some distribution $\rho\left(\left\{X_{i \alpha}\right\}\right)$ that depends on conditions of system's freezing. The two-site cluster approximation, which is used below, is sensitive to the following lowest moments of the distribution $\rho\left(\left\{X_{i \alpha}\right\}\right)$

$$
\left\langle X_{i \alpha}\right\rangle_{x}=c_{\alpha},\left\langle X_{i \alpha} X_{j \beta}\right\rangle_{x}=w_{\alpha \beta}\left(j \in \pi_{i}\right),
$$

where $c_{\alpha}$ is a concentration of the sort $\alpha$ spins.

\section{Formulating a two-site cluster approximation}

The equation (2.8) is quite intractable. One can expand the $\left\langle\tanh \beta \varepsilon_{i}\right\rangle_{H, t}$ in the right-hand side of (2.8) and find that this equation couples correlation functions (CFs) of the type $\left\langle S_{i_{1}} \ldots S_{i_{n}}\right\rangle_{H, t}$ where the site indices belong to the first coordination sphere of the site $i\left(i_{1} \cdots i_{n} \in \pi_{i}\right)$. The equation for $\left\langle S_{i_{1}} \ldots S_{i_{n}}\right\rangle_{H, t}$ derived from (2.4) and (2.5) involves higher CFs, so the exact treatment of the Glauber's equations has succeeded only in the case of one-dimensional system in the zero field (ideal (one-sort) chain $[1,4,5]$, the chain with single impurity [6]). In other cases interpolative approximations are used which express the higher CFs via the lower ones, e.g. $\left\langle\prod_{j} S_{j}\right\rangle_{H, t} \approx \prod_{j}\left\langle S_{j}\right\rangle_{H, t}$.

Here we shall use the closure to the equation (2.8) in the spirit of a cluster approach. In a one-site approximation we replace contributions of all spins with effective fields

$$
\varepsilon_{i} \rightarrow \varepsilon_{i}^{[1]}=\bar{\kappa}_{i, t}+\sum_{r \in \pi_{i}} r \bar{\varphi}_{i, t} \equiv \bar{\kappa}_{i, t}
$$


where ${ }_{r} \bar{\varphi}_{i \alpha}$ has the meaning of effective field acting on the spin $i$ of sort $\alpha$ from the nearest neighbour on the site $r$. In a two-site approximation a contribution of one of the nearest neighbours is taken into account explicitly

$$
\varepsilon_{i} \rightarrow \varepsilon_{i}^{[2]}=\bar{\kappa}_{i, t}+\sum_{\substack{r \in \pi_{i} \\ r \neq 2}} \bar{\varphi}_{i, t}+K_{i j} S_{j} \equiv{ }_{j} \bar{\kappa}_{i, t}+K_{i j} S_{j}, \quad\left(j \in \pi_{i}\right) .
$$

The fields ${ }_{r} \bar{\varphi}_{i \alpha}$ have to be found assuming that the results of one-site and two-site approaches coincide. In the case of ideal (one-sort) system no more assumptions are needed to obtain the closed set of equations for the average value of spin $\left\langle S_{i}\right\rangle_{H, t}$. For the many-sort system it is necessary to point out a dependence of the fields ${ }_{r} \bar{\varphi}_{i, t}$ on the sort configuration. We shall believe the fields ${ }_{r} \bar{\varphi}_{i t}$ to be different in the one-site approximations and in the two-site ones until the averaging over sort configurations is carried out. For the sort averaging procedure both "one-site" and "two-site" fields will be assumed to be locally sort-dependent

$$
{ }_{r} \bar{\varphi}_{i, t}=\sum_{\alpha} X_{i \alpha r} \bar{\varphi}_{i \alpha, t}
$$

where ${ }_{r} \bar{\varphi}_{i \alpha, t}$ does not contain spin or sort variables. In the final expressions the uniform externa field should be assumed $\kappa_{i \alpha} \rightarrow \kappa_{\alpha}$. After such approximations it is a matter of rather tedious but straightforward calculations to obtain the equations for the static part of the mean spin value and the dynamic correlation function of the model $m^{(2)}(\vec{q}, \omega)=\sum_{j} \exp \left(\mathrm{i} \vec{q} \vec{R}_{j}\right) \delta m_{i \alpha}^{(1)}(\omega) / \delta \kappa_{j \beta}(\omega)[3]$.

In figure 1 the results of the theory are compared with dielectric measurements for $\mathrm{Cs}\left(\mathrm{H}_{1-x} \mathrm{D}_{x}\right)_{2} \mathrm{PO}_{4}$ crystals $[7,8]$. At given model parameters the theory predicts almost monodispersive relaxation in paraphase for all $x$. Such behaviour actually takes place in the experiment for $\operatorname{Cs}\left(\mathrm{H}_{1-x} \mathrm{D}_{x}\right)_{2} \mathrm{PO}_{4}$. Figure 1 shows, that in the case of the quenched disorder with complete sort chaos $\left(w_{\alpha \beta}=c_{\alpha} c_{\beta}\right)$ the static permittivity differs from that of $\mathrm{Cs}\left(\mathrm{H}_{1-x} \mathrm{D}_{x}\right)_{2} \mathrm{PO}_{4}$. Therefore we have rejected these (quite artificial) restrictions and obtained a good fit to experiment. Model predicts sort ordering in the system: great values of $w_{12}$ signify that hydrogens and deuterons tend to be positioned at the nearest neighbour H-bonds. It means that the following ordering of $\mathrm{H}$ and $\mathrm{D}$ ions in chains takes place: DHDDDHDDHDHD at $c_{\mathrm{H}}<c_{\mathrm{D}}$ (protons never meet together), HDHHDHHHHDHDH at $c_{\mathrm{H}}>c_{\mathrm{D}}$ (deuterons are never positioned at neighbouring hydrogen bonds) and DHDHDH at $c_{\mathrm{H}}=c_{\mathrm{D}}=\frac{1}{2}$.

\section{Mitsui model}

There exist ferroelectric crystals of order-disorder type with asymmetric doublewell potential which have a set of rather unusual properties. In particular, they are the crystals such as Rochelle Salt (Rs), RbHSO $4, \mathrm{NH}_{4} \mathrm{HSO}_{4},\left(\mathrm{NH}_{4}\right)_{3} \mathrm{H}\left(\mathrm{SO}_{4}\right)_{2}$. For the detailed information on the structure and current notion about mechanism of phase transitions in Rochelle Salt the reader is referred to [9]. The Rochelle Salt crystals $\left(\mathrm{NaKC}_{4} \mathrm{H}_{4} \mathrm{O}_{6} \cdot 4 \mathrm{H}_{2} \mathrm{O}\right)$ have two transition temperatures. Ferroelectric phase occurs between $T_{\mathrm{c} 2}=255 \mathrm{~K}$ and $T_{\mathrm{c} 1}=297 \mathrm{~K}$ (in deuterated crystal $T_{\mathrm{c} 2}=251 \mathrm{~K}$ and 


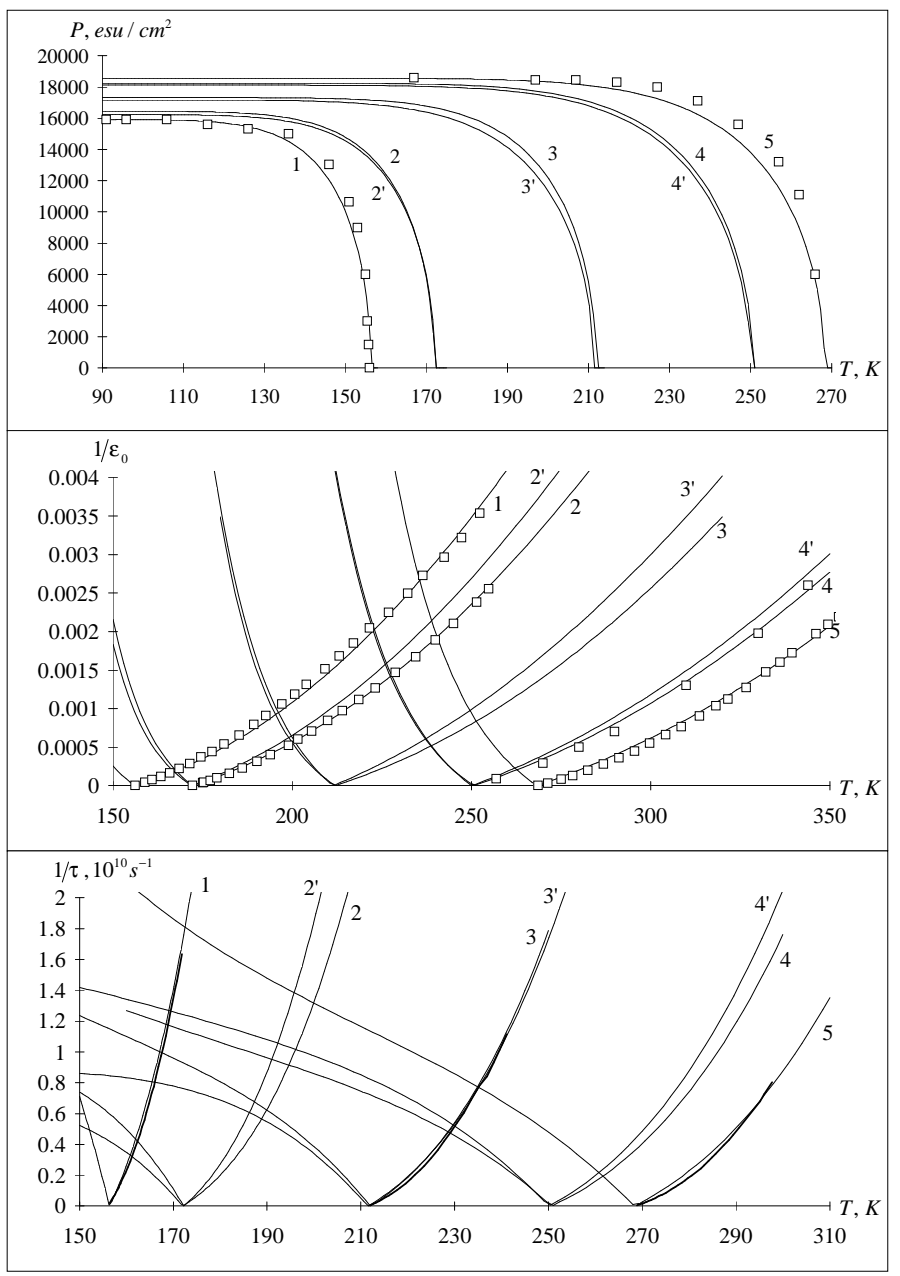

Figure 1. Spontaneous polarization $P$, inverse dielectric permittivity $1 / \varepsilon_{0}$ and inverse relaxation time $1 / \tau$ of the ferroelectric $\mathrm{Cs}\left(\mathrm{H}_{1-x} \mathrm{D}_{x}\right)_{2} \mathrm{PO}_{4}$ at different degrees of deuteration. Squares correspond to experimental data [7], thin lines in the lower picture connect experimental points from the paper [8]. Thick lines, denoted by numbers, present theoretical results for the model for concentrations $x=0,0.2,0.54,0.88,1$. In the case of the complete sort chaos: $w_{\alpha \beta}=c_{\alpha} c_{\beta}$ (lines 2', 3', 4') there is no satisfactory agreement between the theoretical and experimental values of $1 / \varepsilon_{0}$. Good fit to experimental data can be reached, if we suppose that deuterons and protons tend to occupy the nearest $H$-bonds: $w_{12}=\min \left(c_{1}, c_{2}\right)>c_{1} c_{2}$ (lines $\left.2-4\right)$.

$\left.T_{\mathrm{c} 1}=308 K\right)$. Both phase transitions are of the second order. It is thought, that the main role in ferroelectricity of Rs is played by the dipole moments of hydroxyl groups and, to some extent, by crystallization water molecules. Their reorientation makes a major contribution to the emerging of spontaneous polarization. These dipoles move in effective asymmetric double-well potentials.

Mitsui model [10] is most successful in microscopic description of Rs [11-15]. In order to describe the effect of deuteration we consider the disordered Mitsui model 

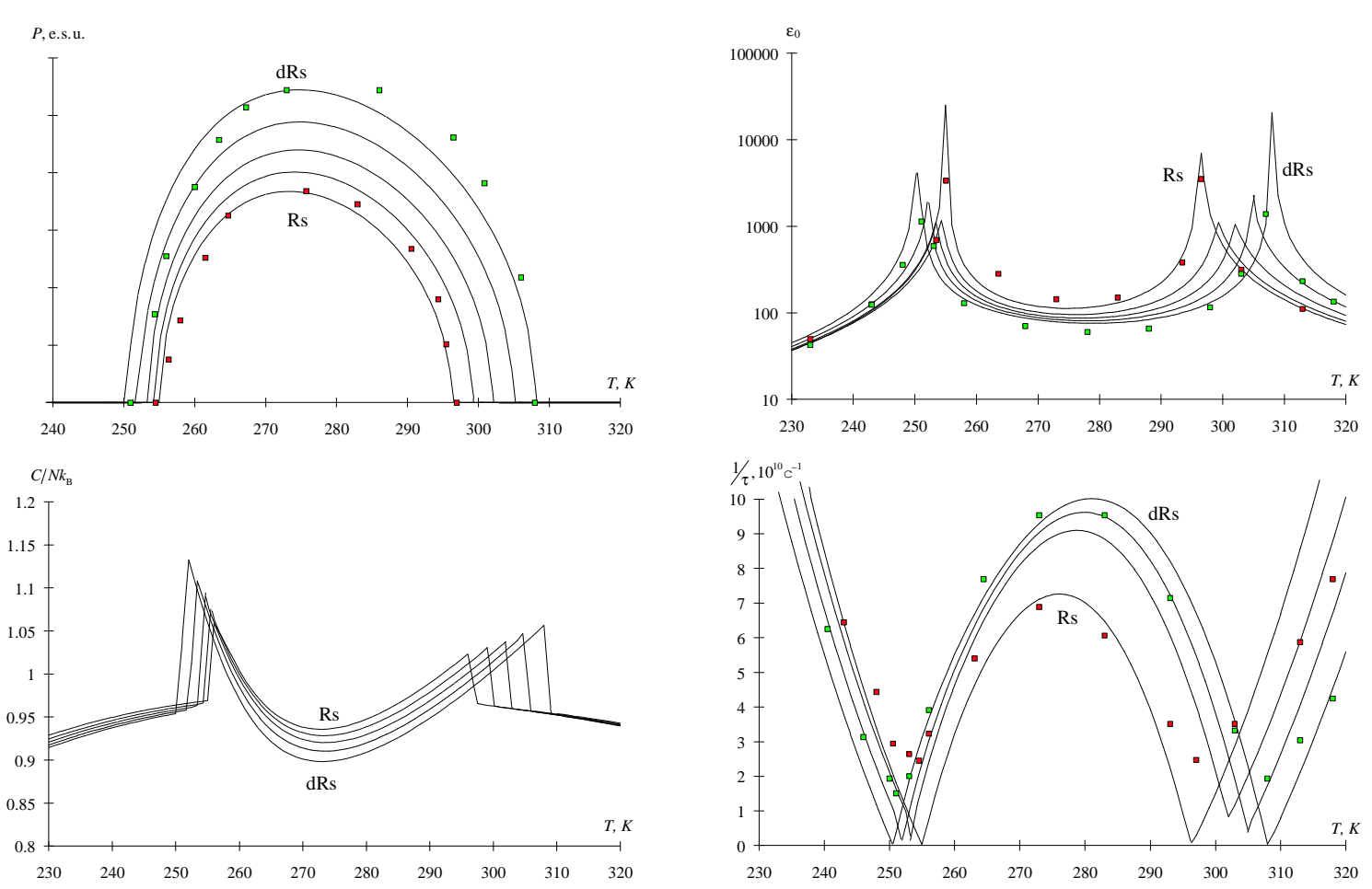

Figure 2. Temperature dependence of spontaneous polarization, spin contribution to specific heat, static dielectric permittivity $\varepsilon_{0}$ and inverse relaxation time $1 / \tau$ of $\mathrm{Rs}_{1-x} \mathrm{dRs}_{x}$ crystals. Lines are theoretical results for the model with parameters given in table 1 ( for $x=0,0.25,0.5,0.75,1$ ), symbols correspond to the results of dielectric measurements [11] for pure crystals $(x=0$ and $x=1)$.

$$
H=-\frac{1}{2} \sum_{\substack{i j \\ a b}} J_{\frac{i}{a} \frac{j}{b}} S_{\frac{i}{a}} S_{\frac{j}{b}}-\sum_{i a} \Gamma_{\frac{i}{a}} S_{\frac{i}{a}}-\sum_{i}\left(\Delta_{\underline{\underline{i}}} S_{\frac{i}{+}}-\Delta_{\underline{\underline{i}}} S_{\underline{\underline{i}}}\right) .
$$

It is a Hamiltonian of the two-sublattice Ising model with internal fields $\Delta_{\underline{i}}$ which describes the asymmetry of one-particle potential. The indices $a$ and $b$ number the sublattices and take the values + and - , according to the sign of internal field $\Delta_{\underline{i}}$ in the sublattice; indices $i, j$ denote the number of the site in its sublattice $(i=1 \ldots \stackrel{a}{N})$. Impurities, partial deuteration of the crystal cause the coupling constant $J$, the field $\kappa$, the asymmetry $\Delta$ to be different at different sites and to depend on the sort configuration

Table 1. Parameters of the ideal (one-sort) model, which provide the best fit of the theory to experimental data. For Rs and dRs crystals holds $J_{++}=J_{--}$.

\begin{tabular}{l|cccccc} 
& $K / k_{\mathrm{B}}, \mathrm{K}$ & $J_{++} / k_{\mathrm{B}}, \mathrm{K}$ & $J_{+-} / k_{\mathrm{B}}, \mathrm{K}$ & $\Delta / k_{\mathrm{B}}, \mathrm{K}$ & $\mu$, e.s.u. & $\tau^{0}, \mathrm{~s}$ \\
\hline Rs & 220.8 & 29.44 & 154.56 & 154.60 & $1.47 \times 10^{-18}$ & $4 \times 10^{-14}$ \\
dRs & 225.0 & 29.06 & 158.44 & 158.38 & $1.61 \times 10^{-18}$ & $5 \times 10^{-14}$
\end{tabular}




$$
J_{\frac{i}{a} \frac{j}{b}}=\sum_{\alpha \beta} J_{\frac{i}{a} \alpha, \frac{i}{b} \beta} X_{\frac{i}{a} \alpha} X_{\frac{j}{b} \beta}, \Gamma_{\frac{i}{a}}=\sum_{\alpha} \Gamma_{\frac{i}{a} \alpha} X_{\frac{i}{a} \alpha}, \Delta_{\frac{i}{a}}=\sum_{\alpha} \Delta_{\alpha} X_{\frac{i}{a} \alpha} .
$$

We account short-range intrasublattice interactions within TCA, while longrange interactions and interactions between spins of different sublattices are taken into account within the mean field approximations. The dynamic pair $\mathrm{CF}$ of the model in such an approach can be expressed via dynamic pair CFs of Ising model (the derivation is similar to that given in the appendix in [3])

$$
\begin{aligned}
\left(\begin{array}{ll}
\hat{M}_{++}^{(2)}(\vec{q}, \omega) & \hat{M}_{+-}^{(2)}(\vec{q}, \omega) \\
\hat{M}_{-+}^{(2)}(\vec{q}, \omega) & \hat{M}_{--}^{(2)}(\vec{q}, \omega)
\end{array}\right)^{-1}= & \left(\begin{array}{cc}
\hat{m}_{++}^{(2)}(\vec{q}, \omega) & 0 \\
0 & \hat{m}_{--}^{(2)}(\vec{q}, \omega)
\end{array}\right)^{-1} \\
& -\beta\left(\begin{array}{cc}
\hat{J}_{++}(\vec{q}) & \hat{J}_{+-}^{(\vec{q})} \\
\hat{J}_{-+}(\vec{q}) & \hat{J}_{--}(\vec{q})
\end{array}\right)
\end{aligned}
$$

where

$$
\begin{aligned}
& \left(\hat{M}_{a b}^{(2)}(\vec{q}, \omega)\right)_{\alpha \beta}=\sum_{j} e^{\mathrm{i}\left(\vec{q}\left(\vec{R}_{i}-\vec{R}_{j}\right)\right.}\left\langle\left\langle S_{\frac{i}{a} \alpha} S_{\frac{j}{b} \beta}\right\rangle_{H, \omega}^{c}\right\rangle_{x}, \\
& \left(\hat{J}_{a b}(\vec{q}, \omega)\right)_{\alpha \beta}=\sum_{j} e^{\mathrm{i} \vec{q}\left(\vec{R}_{i}-\vec{R}_{j}\right)} J_{\frac{i}{a} \alpha, \frac{i}{b} \beta}
\end{aligned}
$$

and $\hat{m}_{a a}^{(2)}(\vec{q}, \omega)$ is a correlation function of Ising model with additional field $-a \Delta_{\frac{i}{a}}$. In figure 2 the results of the theory and experimental data on Rs and dRs crystals are compared. Since there are no experimental data on partially deuterated Rs crystals, we can not check the validity of our theory for disordered crystals. In the figure theoretical lines for several degrees of deuteration are presented for the case of complete sort chaos $w_{12}=c_{1} c_{2}$ and dipole-dipole type of interaction between sorts: $K_{\mathrm{HD}}=\sqrt{K_{\mathrm{HH}} K_{\mathrm{DD}}}, J_{a \mathrm{HbD}}=\sqrt{J_{a \mathrm{H} b \mathrm{H}} J_{a \mathrm{D} b \mathrm{D}}}\left(J_{a \alpha b \beta}=\sum_{j} J_{\frac{i}{a} \alpha \frac{j}{b} \beta}\right)$.

\section{References}

1. Glauber R.J. Time-dependent statistics of the Ising model. // J. Math. Phys., 1963, vol. 4, No. 2, p. 294-307.

2. Levitskii R.R., Sorokov S.I., Sokolovskii R.O. Correlation functions of quenched and annealed Ising systems. // Cond. Matt. Phys., 1995, No. 5, p. 81-104.

3. Levitskii R.R., Sorokov S.I., Sokolovskii R.O. Relaxation dynamics of disordered Ising model. Two-site cluster approximation. // Cond. Matt. Phys. 1996, No. 6, p. 7117-132.

4. Suzuki M., Kubo R. Dynamics of the Ising model near the critical point. I. // J. Phys. Soc. Japan, 1968, vol. 24, No. 1, p. 51-60.

5. Hilhorst H.J., Suzuki M., Felderhof B.U. Kinetics of the stochastic Ising chain in a two-flip model. // Physica, 1972, vol. 60, p. 199-222.

6. Calef D.F. Impurity dynamics in a one-dimensional chain. // J. Stat. Phys., 1983, vol. 32, No. 1, p. 81-94.

7. Deguchi K., Okaue E., Nakamura E. Effect of deuteration on the dielectric properties of ferroelectric $\mathrm{CsH}_{2} \mathrm{PO}_{4}$. I. Static dielectric properties. // J. Phys. Soc. Jpn., 1982, vol. 51 , No. 11 , p. $3575-3582$. 
8. Deguchi K., Nakamura E., Okaue E., Aramaki N. Effect of deuteration on the dielectric properties of ferroelectric $\mathrm{CsH}_{2} \mathrm{PO}_{4}$. II. Dynamic dielectric properties. // J. Phys. Soc. Jpn., 1982, vol. 51, No. 11, p. 3583-3590.

9. Iona F., Shirone D. Ferroelectric crystals. Moscow, Mir, 1965 (In Russian).

10. Mitsui T. Theory of the ferroelectric effect in rochelle salt. // Phys. Rev., 1958, vol. 111, No. 5, p. 1529-1567.

11. Sandy F., Jones R.V. Dielectric relaxation in Rochelle salt. // Phys. Rev., 1968, vol. 168 , p. $4481-4493$

12. Zeks B., Schukla L.C., Blinc R. Dynamics of ferroelectric rochelle salt. // Phys. Rev., 1971, vol. 3, No. 7, p. 2305-2311.

13. Vaks V.G. Introduction to microscopic theory of ferroelectrics. Moscow, Nauka, 1973 (In Russian).

14. Kalenik J. Pseudospin model for the ferroelectric Rochelle salt in the molecular field approximation.. // Acta Phys. Pol., 1975, vol. A48, No. 3, p. 387-395. (In Russian)

15. Levitskii R.R., Antoniak Yu.T., Zachek I.R. Relaxational phenomena in deuterated Rochelle Salt.. // Ukr. Fiz. Zhourn., 1981, vol. 26, No. 11, p. 1835-1838.

16. Grigas Y., Zachek I.R., Krasikov V.C., Kutny I.V., Levitskii R.R., Paprotny V. Isotopic effect in $\mathrm{RbHSO}_{4} .$. // Lit. Fiz. Sbornik, 1984, vol. 24, No. 6, p. 33-45. (In Russian).

\section{Релаксаційна динаміка невпорядкованих ізінгівських моделей}

\section{Р.Р.Левицький, Р.О.Соколовський}

Інститут фізики конденсованих систем НАН України, 79011 Львів, вул. Свєнціцького, 1

\section{Отримано 11 жовтня 1998 р.}

Розглядається невпорядкована модель сегнетоелектриків типу порядок-безпорядок з асиметричним двомінімумним потенціалом. Для випадків рівноважного і нерівноважного безладу розраховані термодинамічні і динамічні характеристики моделі в наближенні двочастинкового кластера за короткосяжною взаємодією з врахуванням далекодії у наближенні молекулярного поля. Модель забезпечує хороший кількісний опис результатів діелектричних вимірювань у кристалах сегнетової солі, гідросульфату рубідію та їх дейтерованих аналогах.

Ключові слова: модель ззінга, модель Міцуї, кінетичне рівняння, сегнетоелектрики типу лад-безлад, динамічна діелектрична проникність

PACS: $64.60 . \mathrm{Cn}$ 